\title{
Direct Observation of Confined Single Chain Dynamics by Neutron Scattering
}

\author{
J. Martín, ${ }^{1}$ M. Krutyeva, ${ }^{2}$ M. Monkenbusch, ${ }^{2}$ A. Arbe, ${ }^{3}$ J. Allgaier, ${ }^{2}$ A. Radulescu, ${ }^{4}$ P. Falus, ${ }^{5}$ J. Maiz, ${ }^{1}$ C. Mijangos, ${ }^{1}$ \\ J. Colmenero, ${ }^{3,6}$ and D. Richter ${ }^{2}$ \\ ${ }^{1}$ Instituto de Ciencia y Tecnología de Polímeros, CSIC, Madrid, Spain \\ ${ }^{2}$ Institut für Festkörperforschung, Forschungszentrum Jülich, Jülich, Germany \\ ${ }^{3}$ Centro de Física de Materiales (CSIC-UPV/EHU), San Sebastián, Spain \\ ${ }^{4} J$ ülich Center of Neutron Science (JCNS) at FRM II, Garching, Germany \\ ${ }^{5}$ Institut Laue-Langevin (ILL), Grenoble, France \\ ${ }^{6}$ Materials Physics Center (MPC) and Donostia International Physics Center, San Sebastián, Spain
}

(Received 4 December 2009; published 11 May 2010)

\begin{abstract}
Neutron spin echo has revealed the single chain dynamic structure factor of entangled polymer chains confined in cylindrical nanopores with chain dimensions either much larger or smaller than the lateral pore sizes. In both situations, a slowing down of the dynamics with respect to the bulk behavior is only observed at intermediate times. The results at long times provide a direct microscopic measurement of the entanglement distance under confinement. They constitute the first experimental microscopic evidence of the dilution of the total entanglement density in a polymer melt under strong confinement, a phenomenon that so far was hypothesized on the basis of various macroscopic observations.
\end{abstract}

DOI: 10.1103/PhysRevLett.104.197801

Confinement effects in polymer melts may lead to unusual properties. This concerns both the chain conformation, which may be distorted, as well as chain dynamics, which may be altered due to surface interactions and changes of topology and chain self-density [1-4]. The understanding of such behavior is not only a scientific challenge but is also important for knowledge-based applications in nanotechnology, such as nanocomposites, coatings, adhesives, etc. [5]. Today, microscopic studies on the chain dynamics under confinement are mainly available through simulations. Only a few experiments have addressed this problem, e.g., the flow of polymers through nanopores, the extensional rheology of nanosized polymer films, the observation of dewetting kinetics of thin films or NMR relaxometry. Chain dynamics is commonly described in terms of the Rouse and the reptation model. The relaxation of the Rouse modes, determined by a balance of viscous and entropic forces, only depends on the chain length and the monomeric friction. In addition, long polymers heavily interpenetrate each other and mutually restrict their motions at long times in forming topological constraints ("entanglements"). In the reptation model the entanglement effect is modeled by a tube of diameter $d \sim$ $\ell \sqrt{N_{e}}$ along the coarse grained chain profile confining the chain motion $\left(\ell\right.$, monomer length; $N_{e}$, number of monomers between the entanglements). The dominant motional mechanisms in this model are (i) a curvilinear version of the Rouse motion (local reptation) followed by (ii) the escape of the whole molecule from the tube at long times, the reptation process (see, e.g., [6,7]). The important question that is addressed now both by simulations $[2,3,8,9]$ as well as by a variety of experiments on a macroscopic level $[4,10-12]$ is how these dynamics change under confinement.
PACS numbers: 61.41.+e, 62.25.- $\mathrm{g}, 78.70 . \mathrm{Nx}, 82.35 . \mathrm{Lr}$

Basically all simulations available indicate that confinement reduces chain mobility independent of the adhesive potential of the wall. An analysis of the Rouse modes of unentangled chains under confinement reveals a uniform slowing down of all modes which was interpreted by an effective increase of monomeric friction [8]. The consequences for the entanglement density are less clear: while in the early work of Shaffer [1] no influence was found, newer simulations $[2,3,9]$ seem to indicate an increase of the entanglement distance that was related to chain compression and thereby to the reduction of the interchain overlap under confinement. The combined effects of increased effective friction and entanglement distances were even found to lead to a nonmonotonic behavior of the chain relaxation with confinement size [2]. Also, heterogeneous scenarios like a repulsion of entanglements away from the confining surface were proposed [9].

Observing a significantly enhanced flow of large polystyrene molecules into the pores of anodic alumina oxide, Russell and co-workers concluded on a reduced viscosity that would follow from a decreasing entanglement density due to lateral chain compression [4]. Performing very delicate studies on the extension ratio of nanoscopic polymer films under shear, $\mathrm{Si}$ et al. also conjectured on a reduced density of interchain entanglements which, according to them, should be compensated by a corresponding increase of intrachain entanglements [10]. Similarly, from dewetting experiments a significantly reduced entanglement density in thin polymer films was conjectured [11]. Finally, an antagonistic effect of confinement was concluded from NMR relaxometry studies, that reported a reduction of the tube diameter in confined polymer melts by about 1 order of magnitude as compared to the bulk ("Corset effect") [12]. 

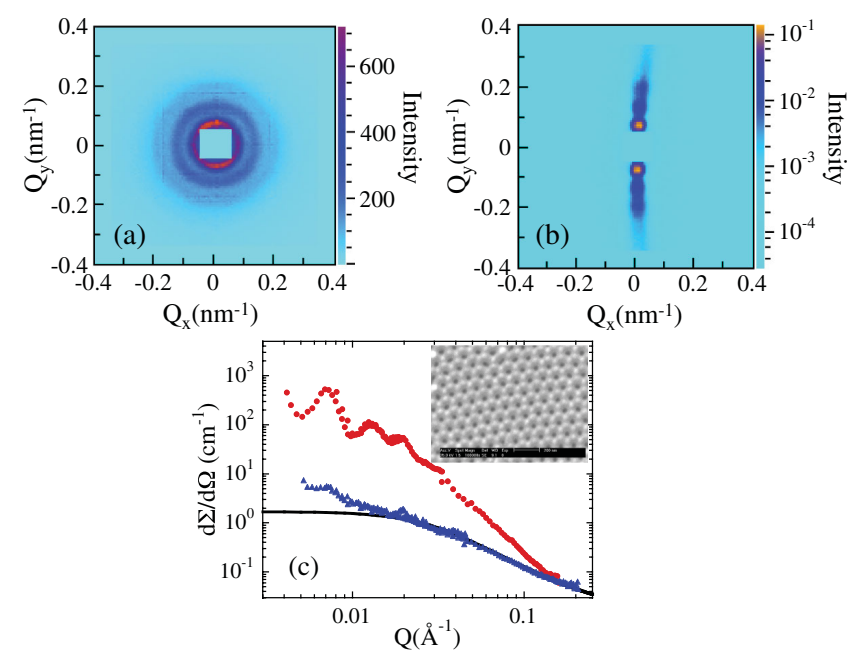

FIG. 1 (color). (a) 2D-SANS pattern of sample I with the pores oriented parallel to the incident beam. The powderlike appearance is due to a resulting random orientation of the hexagonal lattice in the stacked templates. (b) The same as (a) but with the sample rotated by $\phi=15^{\circ}$ around the vertical axis. (c) Cross sections obtained for $\phi=15^{\circ}$ [data in (b)] calculated by radial averaging of $2 \mathrm{D}$ pattern (circles) and integrated along the horizontal direction (triangles). Solid line: Debye form factor with $R_{g}=5.5 \mathrm{~nm}$. Rocking measurements showed that for $\phi \geq 10^{\circ}$ the template's contribution in the NSE window is roughly constant. Inset: Scanning electron micrograph (Philips XL-30 ESEM) of the surface of the AAO templates.

Using the space-time resolution of neutron spin echo spectroscopy (NSE) [7], here we report the first direct observation of the entanglement distances and the associated intermediate scale Rouse dynamics under confinement conditions. We present results on the single chain dynamic structure factor of poly(ethylene oxide) (PEO) confined in cylindrical nanopores of anodic alumina oxide (AAO). The chain dimensions (average bulk end-to-end distance $R_{e}=\sqrt{\left\langle R_{e}^{2}\right\rangle}$ ) were either much larger or smaller than the lateral pore sizes (characterized by the pore diameter $D$ ). At long times well beyond the entanglement limit, for $R_{e}>D$ we found a $15 \%$ expanded entanglement network, while for $R_{e}<D$ the bulk values are reproduced. As compared to bulk PEO, a moderate slowing down of the dynamics in the intermediate time regime was noticed. This effect, other than simulations suggested, may not be related to a general increase of the Rouse friction, since the local dynamics at short times is found to be largely unchanged.
Ordered AAO templates were prepared by a two-step anodization process of aluminum [13]. The templates consist of a hexagonal array of parallel cylindrical pores of $120 \mu \mathrm{m}$ depth oriented perpendicularly to the surface of the template [inset of Fig. 1(c)]. Two samples were prepared with different $R_{e} / D$ ratios (see Table I). The nanoporous channels were filled with mixtures of deuterated $(d)$ and protonated $(h)$ PEO chains in fractions of $0.748 / 0.252(\mathrm{v} / \mathrm{v})$. Such mixtures have the same neutron scattering-length density as the templates, that had been previously determined by a contrast matching small angle neutron scattering (SANS) experiment with dioxane. Prior to the NSE experiments, we checked the quality of the matching on sample I at $400 \mathrm{~K}$ at the SANS instrument KWS-2 (JCNS at FRM-II Munich). The results obtained with pore orientation parallel to the incident beam are displayed in Fig. 1(a). The AAO scattering intensity is confined to a narrow sheet in reciprocal space perpendicular to the pore direction. For SANS the curvature of the Ewald sphere is very small, the intensity sheet is tangential to the detected patch, and SANS reveals the pore arrangement in an imperfect lattice. Figure 1(a) shows that, though matching reduced the pore scattering intensity considerably, it is still visible. Tilting the sample a given angle $\phi$ around the vertical axis, the cut of the inclined intensity sheet on the Ewald sphere becomes a circle, and any residual scattering from the membrane occurs on the vertical axis $\left(Q_{x}=0\right)$ [see Fig. 1(b)]. Figure 1(c) shows with triangles the cross section integrated along the horizontal direction in the 2D pattern of Fig. 1(b) and the calculated single chain form factor [solid line, Debye function $P(x)=$ $\left(e^{-x}+x-1\right) / x^{2} ; x=Q^{2} R_{g}^{2}$ ] with the bulk value for the radius of gyration $R_{g}$. For $Q$ values above $\approx 10^{-2} \AA^{-1}$ the agreement is excellent. Thus, with such geometry and for $Q>10^{-2} \AA^{-1}$, the intensity scattered in the horizontal direction is mainly due to the contrast between deuterated and protonated polymer chains. There, SANS reveals the single chain structure factor and NSE accesses the single chain dynamic structure factor $S_{\text {chain }}(Q, t)[7,14]$ of the confined polymer mixture.

The dynamic experiments on sample I were performed at the JCNS spectrometer J-NSE at the FRM II and on sample II at the IN15 instrument at the ILL (Grenoble). Different $Q$ values were investigated at $400 \mathrm{~K}$ in the Fourier time intervals $0.2 \leq t \leq 130 \mathrm{~ns}$ (J-NSE) and $9 \mathrm{ps} \leq t \leq 200 \mathrm{~ns}$ (IN15) by combining two incident wavelengths $(\lambda=8$ and $12.9 \AA$ at J-NSE, $\lambda=8$ and $16 \AA$ at IN15). Because of the small amount of polymer

TABLE I. Details of the samples investigated.

\begin{tabular}{|c|c|c|c|c|c|c|c|c|c|}
\hline Sample & $M_{w}^{h \text { PEO }}(\mathrm{g} / \mathrm{mol})$ & $M_{w} / M_{n}{ }^{\mathrm{a}}$ & $M_{w}^{d \mathrm{PEO}}(\mathrm{g} / \mathrm{mol})$ & $M_{w} / M_{n}$ & Number of templates & Polymer mass $(M)(\mathrm{g})$ & $R_{e}(\mathrm{~nm})$ & $D(\mathrm{~nm})$ & $R_{e} / D$ \\
\hline I & 22700 & 1.01 & 19500 & 1.02 & 30 & 0.54 & 13.5 & 35 & 0.4 \\
\hline II & 480000 & 1.07 & 430000 & 1.2 & 15 & 0.39 & 59.7 & 25 & 2.4 \\
\hline
\end{tabular}

${ }^{a}$ After release from the nanoconfinement, for sample II somewhat broadened $M_{w}$ distributions were found, indicating some chain rupture that however did not alter the nature of the highly entangled melt. 
in the beam ( $M$ in Table I) measurement times per run were of about $20 \mathrm{~h}$ (J-NSE) and $12 \mathrm{~h}$ (IN15).

Figure 2 shows the results obtained for sample I [2(a)] and sample II [2(b)]. In both cases the data tend to finite plateaus that reflect confinement effects on the dynamics of the polymer chains - the single chain fluctuations are laterally restricted giving rise to a Debye-Waller-type form factor. This is an experimental observation yielding the spatial extent of the molecular motion independent of any model considered. This kind of behavior is also present in the entangled bulk sample [15], where chain confinement is due to topological interactions with the surrounding chains. The bulk results could be well described by the reptation model (see, e.g., [6,7]):

$$
\begin{aligned}
\frac{S(Q, t)}{S(Q)}= & {\left[1-\exp \left(-\frac{Q^{2} d^{2}}{36}\right)\right] S^{\mathrm{loc}}(Q, t) } \\
& +\exp \left(-\frac{Q^{2} d^{2}}{36}\right) S^{\mathrm{esc}}(Q, t),
\end{aligned}
$$

where $S^{\text {loc }}(Q, t)$ and $S^{\text {esc }}(Q, t)$ are contributions from local motion and escape from the tube, respectively. For PEO at $400 \mathrm{~K}, S^{\mathrm{esc}}(Q, t)=1$ in the NSE window and the value of $d$ determines the plateau levels. The contribution of local reptation in the NSE window is given by

$$
S^{\mathrm{loc}}(Q, t)=\exp \left(\frac{t}{\tau_{0}}\right) \operatorname{erfc}\left(\sqrt{\frac{t}{\tau_{0}}}\right),
$$

where $\tau_{0}=36 /\left(W \ell^{4} Q^{4}\right)$ is the time scale for Rouse seg-
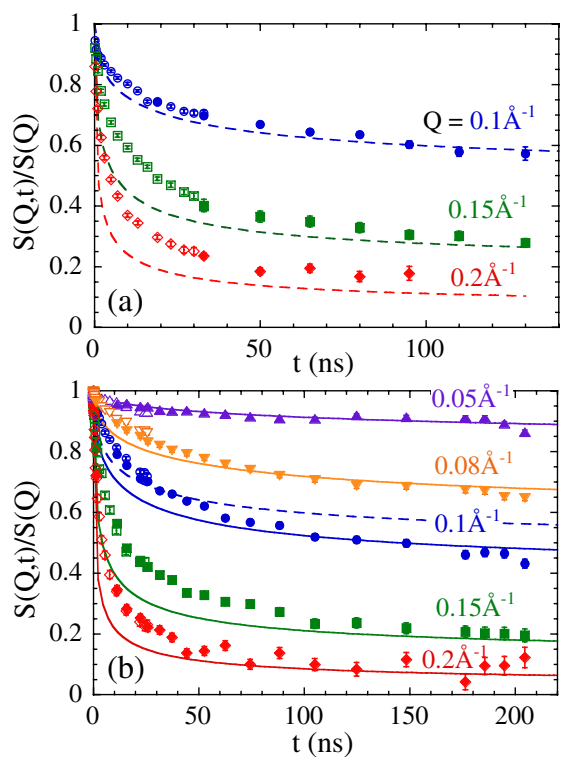

FIG. 2 (color online). Normalized $S_{\text {chain }}(Q, t)$ of the short confined chains (sample I) (a) and the long confined chains (sample II) (b) at the $Q$ values indicated. Empty symbols correspond to data obtained with $\lambda=8 \AA$, full symbols with $\lambda=12.8 \AA \quad$ (J-NSE) and $\lambda=16 \AA \quad$ (IN15). Dashed lines: de Gennes curves [Eq. (1)] fitting the bulk PEO behavior [15]. Solid lines in (b): Fits to Eq. (1) with $W=W^{\text {bulk }}$, delivering a $d^{\text {conf }}$ value of $60.3 \AA$. ment diffusion along the tube with $W$ the Rouse rate (determined by the friction with the medium). For bulk PEO at $400 \mathrm{~K}$, with $\ell=5.8 \AA, W^{\text {bulk }}=13.34 \mathrm{~ns}^{-1}$ and $d^{\text {bulk }}=52.6 \AA$ [15]. The curves describing $S_{\text {chain }}(Q, t)$ of bulk PEO at the corresponding $Q$ values are shown in Fig. 2(a) as dashed lines. They reproduce very well the plateau levels of the confined short-chain polymer. The situation is different for the more confined long chains. In Fig. 2(b), the dashed line shows the de Gennes description of the bulk results for $Q=0.1 \AA^{-1}$. The confined $S_{\text {chain }}(Q, t)$ at this $Q$ decays to significantly lower values, indicating a less restricted dynamics (larger effective tube diameter)

On the other hand, the data show a clear slowing down compared to the bulk behavior at intermediate time scales [see Figs. 2 and 3], while at short times $(t \lesssim 1 \mathrm{~ns})$ the dynamics equals that of the bulk (see Fig. 3). This observation is fully consistent with our previous results on the local dynamics of sample I [16], where quasielastic neutron spectra with resolution of the order of a nanosecond did not reveal essential deviations from the bulk segmental dynamics at length scales smaller than $1.5 \mathrm{~nm}$. Our experiments suggest a higher impact of confinement on the longer-wavelength Rouse modes than on the local scale. This is in contradiction to simulations that were interpreted in terms of an overall slowing down of all Rouse modes [8]. One possible explanation for the observed behavior could be some adsorption effect on the surface of the pore walls. The alumina surface "attracts" hydrophilic PEO chains, leading to a complete wetting of the AAO surface by the polymer melt. In a first approximation the adsorption effect was modeled by a Rouse chain with pinned segments, such that on average the surface layer is covered. Averaging over all positions of the pinned segments along the affected chains the $S_{\text {chain }}(Q, t)$ shown in Fig. 3 are obtained. At short times this simple model reproduces

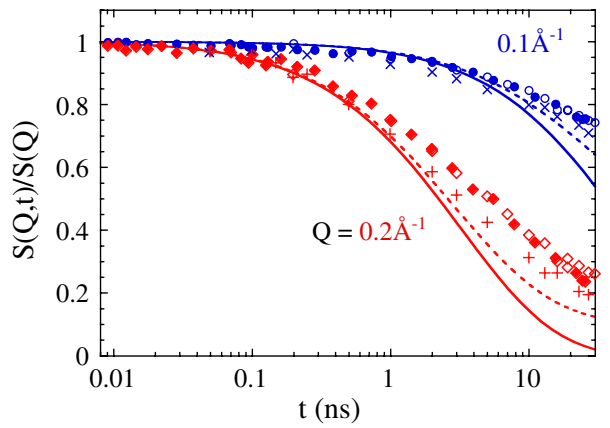

FIG. 3 (color online). NSE data at $Q=0.1 \AA^{-1}$ (circles) and $Q=0.2 \AA^{-1}$ (diamonds) for the short (empty symbols) and long (full symbols) PEO chains compared with the bulk data (crosses and pluses) [15]. Solid lines: Rouse curves displaying the bulk behavior below $1 \mathrm{~ns}$. Dotted lines: Calculated Rouse $S_{\text {chain }}(Q, t)$ for pinned chains. Note that the upturn of the experimental data is due to both the entanglements (see bulk behavior) as well as to the suggested pinning (pore data). 
the unchanged Rouse dynamics, and at longer times it displays the observed tendency.

As previously mentioned, our results provide the definite microscopic proof that under confinement the lateral tube size is only marginally affected. The weakly confined sample shows bulklike entanglement density, while the strongly confined long chains display tube dilation. To quantify this effect, we have fitted the de Gennes equation (1) to the NSE data of sample II imposing the value of $W^{\text {bulk }}$ (which perfectly describes the short-time regime). The fitting curves are shown as solid lines in Fig. 2(b). A value of $d^{\text {conf }}=60.3 \AA$ is obtained; i.e., an increase of about $15 \%$ is observed for a confinement characterized by $R_{e} / D=2.4$ (i.e., $D \approx R_{g}$ ).

Let us now discuss the simulation predictions in light of our microscopic results. Our main observation contradicts an insensitivity of the entanglement network upon confinement as found in Ref. [1]. Our results qualitatively support the competing situation predicted in Ref. [2], where the slowed-down chains experience at the same time confinement-induced disentanglement effects. We note in passing that the good description of the long-time behavior of the experimental $S_{\text {chain }}(Q, t)$ with only one tube diameter is in contradiction with a markedly heterogeneous distribution of entanglement lengths, as suggested for polymer nanocomposites [9]. Finally, good agreement is found with the results by Baschnagel and co-workers [3], who reported a $32 \%$ enhancement of the entanglement length (equivalent to an increase of $15 \%$ in the tube diameter) for thin films with a similar confinement size. We recall, however, that in their case the 1D confinement could be expected to have a lower impact in the dynamics than the 2D confinement imposed in this work.

We now compare our microscopic results with the more macroscopic data published so far. Russell and co-workers [4] reported a decrease of the viscosity with respect to the bulk value by a factor of $\approx 1000$ in the capillary rise of chains in a membrane with $R_{e} / D \approx 3.4$ and $\approx 500$ for $R_{e} / D \approx 2.5$. The corresponding derived tube diameter $\left[d^{\operatorname{conf}}\left(R_{e} / D \approx 2.4\right) / d^{\text {bulk }} \approx 5\right.$ in Ref. [4]] is apparently incompatible with the value of 1.15 from our experiments. We could reconcile both observations in two ways. (i) The flow experiment corresponded to a nonequilibrium situation, and a strict direct comparison of values might not be possible. (ii) Flow experiments are expected to be mainly sensitive to interchain entanglements, the density of which is drastically reduced under strong confinement, while the total entanglement density observed by NSE would be only slightly reduced with respect to the bulk [17]. Also, the proposed reduction (factor 3-4) of the entanglement density based on dewetting experiments [11] is much larger than what is observed on the chain level. Possibly similar arguments hold as compared to the flow experiments. We also mention that the distinction between self-chain and interchain entanglements in confined polymers was an essential ingredient to interpret the extensional strain results in thin polymer films by Si et al. [10]. Their model based on chain packing assumed that the total entanglement density is constant when applied to systems with $R_{e} \lesssim 2 h$ ( $h$, film thickness). With our direct experiments under $2 \mathrm{D}$ confinement we find that for $R_{e}=2.4 \mathrm{D}$ it decreases by about $15 \%$. Our observation could thus be considered in order to improve the model proposed in that work when applied to stronger confinement situations. Finally, the drastic increase of the entanglement density concluded from NMR relaxometry (Corset effect) is out of the question.

We thank M. Hernández-Vélez, A. R. González-Elipe, J. Gil, R. Sastre, O. García, and M. Brodeck, SoftComp NoE, MAT2007-63681, IT-436-07 (GV), the MEyC (CSD2006-53), Grants FPU AP2005-1063 and MAT2008-1073.

[1] S. Shaffer, Macromolecules 29, 1010 (1996).

[2] Y. Li, D. Wei, C. C. Han, and Q. Liao, J. Chem. Phys. 126, 204907 (2007).

[3] H. Meyer, T. Kreer, A. Cavallo, J.P. Wittmer, and J. Baschnagel, Eur. Phys. J. Special Topics 141, 167 (2007).

[4] K. Shin, S. Obukhov, J.-T. Chen, J. Huh, Y. Hwang, S. Mok, P. Dobriyal, P. Thiyagarajan, and T. P. Russell, Nature Mater. 6, 961 (2007).

[5] Y. Mai and Z. Yu, Polymer Nanocomposites (CRC, Boca Raton, FL, 2006).

[6] M. Doi and S. Edwards, The Theory of Polymer Dynamics (Clarendon, Oxford, 1986).

[7] D. Richter, M. Monkenbusch, A. Arbe, and J. Colmenero, Adv. Polym. Sci. 174, 1 (2005).

[8] P. J. Dionne, R. Ozisik, and C. R. Picu, Macromolecules 38, 9351 (2005).

[9] R. A. Riggleman, G. Toepperwein, G. J. Papaconstantopoulos, J.-L. Barrat, and J. J. de Pablo, J. Chem. Phys. 130, 244903 (2009).

[10] L. Si, M. V. Massa, K. Dalnoki-Veress, H. R. Brown, and R. A. L. Jones, Phys. Rev. Lett. 94, 127801 (2005).

[11] O. Bäumchen, R. Fetzer, and K. Jacobs, Phys. Rev. Lett. 103, 247801 (2009).

[12] N. Fatkullin, R. Kimmich, E. Fischer, C. Mattea, U. Beginn, and M. Kroutieva, New J. Phys. 6, 46 (2004).

[13] H. Masuda and K. Fukuda, Science 268, 1466 (1995).

[14] In the $Q$ region explored, the incoherent signal from the protonated chains is relatively small compared to the coherent contribution from $S_{\text {chain }}(Q, t)$. Moreover, NSE reduces the incoherent intensity by $1 / 3$.

[15] K. Niedzwiedz, A. Wischnewski, W. Pyckhout-Hintzen, J. Allgaier, D. Richter, and A. Faraone, Macromolecules 41, 4866 (2008).

[16] M. Krutyeva, J. Martín, A. Arbe, J. Colmenero, C. Mijangos, G. Schneider, T. Unruh, Y. Su, and D. Richter, J. Chem. Phys. 131, 174901 (2009).

[17] We cannot discard that the intrachain entanglement density decreases with time. 